\title{
OLDER ADULTS' ATTITUDES TO FOOD AND NUTRITION: A QUALITATIVE STUDY
}

\author{
J.E. Winter, S.A. McNaughton, C.A. Nowson
}

\begin{abstract}
Objective: To explore the factors that influence food choices of older adults and identify potential sources of dietary advice. Design: A qualitative research design using semi-structured, one on one interviews. Setting: A general medical practice in Victoria, Australia. Participants: Twelve community dwelling adults aged 75 to 89 (mean $82.8 \pm 4.4$ ) years, 92\% living alone and 92\% female. Measurements: Interview questions addressed usual daily food pattern, shopping routines, appetite, importance of diet and potential sources of dietary advice or assistance. Results: Thematic analysis identified key themes influencing food choices were maintaining independence; value of nutrition; childhood patterns; and health factors. Dietary restrictions and concerns with weight gain were expressed, and although these were managed independently, the GP was identified as the first source of information if required. Conclusion: This sample of older adults placed high value on eating well as they age, however a number followed self-imposed dietary restrictions which have the potential to compromise their nutritional status as dietary requirements change. Further research is needed into how to communicate changing nutritional needs to this group.
\end{abstract}

Key words: Elderly, attitudes, nutrition, interviews.

\section{Introduction}

Older adults are at risk of under-nutrition due to normal physiological changes combined with alterations in food choice, food access and health conditions $(1,2)$. Nutritional studies have shown that older adults tend towards consuming a lower energy intake (3), smaller meals, slower eating and reduced physical activity (4). Australian data indicate that adults aged over 70 years consume less energy than younger adults and are less likely to meet requirements for protein, riboflavin and vitamin B6 (5).

Factors that impact on food choice and meal patterns have reported to include social isolation (6), presence of chronic disease resulting in dietary restrictions (7), and difficulties with activities of daily living (ADLs) $(8,9)$. Changes to food intake and the consequent impact on nutritional status can result in increased risk of frailty and reduced functional capabilities $(10,11)$.

Prevalence of malnutrition or nutritional risk amongst older adults in the community has been reported at between $16 \%$ and $43 \%(12,13)$. Although it is recognised

Centre for Physical Activity and Nutrition, School of Exercise and Nutrition Sciences, Deakin University, Burwood, Victoria, Australia

Corresponding Author: J. E. Winter, Centre for Physical Activity and Nutrition, School of Exercise and Nutrition Sciences, Deakin University, Burwood, Victoria, Australia, jane.winter1@au.nestle.com that early identification of nutritional issues is important in preventing nutritional decline, (14) older adults can be resistant to dietary interventions. For example, studies in community-based seniors in Australia has shown low uptake of dietetic referrals and resistance to a home delivered meal intervention $(15,16)$, however it is not clear what sources of information older adults do use, if any, to make decisions regarding diet or food choice.

General practitioners (GPs) and other primary health staff such as nurses, have been identified as preferred providers of nutritional care providing trustworthy and personalised care (17), however a study of older adults aged 75 years and over suggested some scepticism about dietary advice provided by GPs (18).

This qualitative study aimed to build on current understanding of food choices of community living older adults and explore potential acceptable sources of nutritional advice and support.

\section{Methods}

Participants were community dwelling adults aged 75 years or older who had a health assessment ("75+ health assessment") within the previous three months and were recruited from a general medical practice in Victoria, Australia. The 75+ health assessment is an annual government funded health assessment offered 
Table 1

Interview questions and inquiry logic

\begin{tabular}{|c|c|}
\hline Inquiry Logic & Interview Question \\
\hline \multirow[t]{4}{*}{ Understand general social \& health situation } & Living arrangements (where, alone, with spouse, with family) \\
\hline & Any assistance with meals \\
\hline & How would you rate your health \\
\hline & Height / weight \\
\hline Understand influences of health status on food choices & Tell me about any health conditions that influence your diet. \\
\hline Explore perception of weight on health & Can you tell me about your current weight? \\
\hline \multirow[t]{4}{*}{ Explore food choices and meal patterns } & What would you usually eat in a typical day: \\
\hline & - Frequency \\
\hline & - Location \\
\hline & - Preparation techniques \\
\hline Understand whether appetite has affected dietary intake & $\begin{array}{l}\text { How would you rate your appetite compared with } 10 \text { years } \\
\text { ago? Describe changes }\end{array}$ \\
\hline Identify issues associated with food access & Tell me about how you do your food shopping. \\
\hline Relative importance or changing role of diet with age. & How do you think dietary needs change with age? \\
\hline Determine sources of advice regarding diet / nutrition & Any dietary advice in the past? \\
\hline
\end{tabular}

to adults aged 75 years or older. Sixty patients who had most recently attended the practice in May 2014 were sent a letter from the practice inviting them to participate in the study.

One on one, semi-structured interviews were conducted by an experienced dietitian (JW). Qualitative inquiry was used as it is well placed to answer complex questions about food behaviours by investigating how and why individuals act in certain ways (19). Openended questions were developed using an inquiry logic that reflected the study aims (Table 1 ). Interview questions addressed usual daily food pattern, shopping routines, appetite, perceived importance of diet and potential sources of dietary advice. Information was also collected on age, living situation, weight, and height. The Mini Nutritional Assessment (MNA®-SF), a validated nutritional screening tool for adults aged 65 years and older, was used to determine nutritional risk of the participants. The MNA ${ }^{\circ}-S F$ comprises six questions about food intake, weight loss, mobility, recent acute illness, cognitive function and body mass index (BMI).The study protocol was approved by Faculty of Health Human Ethics Advisory Group on behalf of the Deakin University Human Research Advisory Committee (HEAG-H 48_2014). All participants provided written informed consent.

Interviews were audio-recorded and transcribed verbatim. Notes were also taken during the interview and compared with the transcripts. Thematic content analysis was used to categorise and codify the interview transcripts $(20,21)$. An inductive thematic analysis was used to identify emergent themes from the data, coding it without trying to fit it into a pre-existing frame (21). Transcripts were read through several times and notes made on general themes and related categories of data. Interviews and analyses were conducted by a single investigator, and a second researcher coded $25 \%$ of the transcripts to verify the coding. Any differences were discussed until agreement was reached. The transcripts were imported into NVIVO 9 (QSR International Pty Ltd), coded according to the initial notes and then categories were collapsed to generate themes for each of the four areas of interest: dietary patterns; influences on food choices; dietary changes with ageing; and sources of dietary advice.

\section{Results}

Of the 60 people invited to participate in the study, 16 contacted the surgery to arrange an interview time. Four later withdrew due to illness (three) or confusion over appointment times (one). Twelve interviews were included in the analysis, at which point data saturation was considered to be reached with no new concepts emerging. Eleven interviews were conducted in a private room at the medical practice, one was conducted at the participant's home at their request. The average interview duration was 33 minutes.

The age of the participants ranged from 75 to 89 years (mean $82.8 \pm 4.4$ years). Eleven participants were female $(92 \%)$, and $11(92 \%)$ lived alone. Three participants 
$(25 \%)$ were classified as being at risk of malnutrition according to the MNA®-SF, all three had suffered acute illness or psychological stress within the previous three months, however all reported that the issues had, or were resolving. No participants were classified as malnourished.

Overall participants felt that they had good, healthy diets and that nutrition was important to their overall health and well-being.

"Very important [diet]. I think particularly when you live on your own, you can get in to really bad habits....but oh yes, it's fundamental isn't it? It's very important." (Female \#11, 75yrs)

"I cook every day. I don't eat junk food. I don't like it." (Female \#2, 83years)

Key themes identified in the analysis are described below under the topics of dietary patterns, food choices, age related change and dietary advice.

\section{Dietary Patterns}

The usual dietary pattern described involved three meals per day, with skipping meals a rare occurrence. As nearly all participants lived alone, most meals were eaten alone in their own homes. Eating out occasions were rare, but more commonly involved meeting friends for 'coffee' or having a cup of tea or coffee, with or without a snack when at the shops.

\section{Routine}

Days tended to be fairly structured with similar meal times each day. There was usually a standard time that participants arose each morning and meals were then organised according to the activities of the day. When describing their meals, it was common to qualify their statements with "every day" or "always". Sometimes these routines reflected long-standing habits.

"I've been doing it for a long time, same old routine so I can't change it" (Female \#5, 86years)

"I still got used to when I worked in the factory 12 o'clock it must be lunch." (Male \#4, 86yrs)

\section{Food Preparation}

As the majority of respondents were female, they had been responsible for food preparation for most of their adult lives, and continued to cook for themselves even when they were living alone. All reported consuming at least one hot meal each day, but often cooked sufficient quantity to last for a few days.

"I'm all for cooking up, you know, larger quantities like that. If I cook a couple of cutlets I'll cook say four, it's two for one night, and an alternate night you have the other two." (Female $\# 7$, 86years)

Despite a desire to prepare their own food, many had started using packaged frozen foods from the supermarket or at least having some in the freezer in case they didn't feel like cooking or had unexpected guests.

"Well, sometimes, I always keep a couple of supermarket, McCain meals in the freezer, in case I'm sick and I can't be bothered by the... I heat up one of those." (Female \#9, 89 years)

\section{Influences on food choices}

\section{Independence and positive attitude}

Participants expressed pride in their ability to remain independent and self-sufficient in all facets of their lives, including shopping and preparing food. They felt that staying active either at home, within their family or with social groups was an important factor in their general health. Even when faced with health issues, they felt that 'just getting on with it' was important.

"I can't do very much. I try, but... and I keep trying til I'm exhausted." (Female \#10, 86 years)

"actually, sometimes I think, when you've got a bit of responsibility, it makes you get up and get going. You can't say, 'Oh, I'll just sit in all day today'." (Female \# 8, 78 years).

\section{Value of eating well}

Diet and nutrition was considered to be important to their overall health, and therefore participants felt it was worth the effort to continue with food preparation.

"I still prepare and cook my own meals.....But, I eat well. I'm a healthy eater." (Female \#9, 89 years)

It was acknowledged that it could be easy to slip into bad habits such as missing meals but the value they placed on diet, prevented this. They often felt that they were doing better than others of their age who appeared to place a lower value on their own well-being.

"always good meals, you know? Yeah, I think it is, because some people say, 'oh, we never cook, eat sandwich'. I don't like that." (Female \# 2, 83 years)

"But she [friend] tells me what she's eating, and she's not eating like I am eating, and you know sometimes, "Oh, I couldn't be bothered making a meal," I would never be like that." (Female \# 1,84 years)

\section{Childhood patterns}

Participants talked about their current food patterns as similar to those they were brought up on and that their parents provided for them. Some food choices were unchanged over many years. The provision of regular 'good' meals as children appeared to set the standard for dietary practices over the course of their adult life.

"well, we were brought up to, on a farm. And my mum and, and dad always made sure we were well fed. And you know we just eat the same. Meat and three veg." (Female \# 9, 89 years) 


\section{Health Conditions}

Food choices were commonly restricted or influenced by health conditions or previous dietary advice. Six of the female participants were conscious of their weight and did restrict food intake to try and reduce their weight. In some instances, this was even in the presence of recent weight loss due to illness or emotional distress.

"I have lost a bit of weight in the last six months, which is part of this [illness] but this is more my natural weight" (Female \# 7,86 years)

Specific foods were often chosen to meet the perceived personal dietary needs or restrictions of participants. Food restrictions included full fat dairy products, artificial preservatives, lactose, fructose and artificial sweeteners. These choices appeared to be self-imposed with little guidance from any health care professionals.

\section{Changes with age}

\section{Inevitability}

Changes associated with age were seen as inevitable and something to be accepted and managed. Participants associated changes to their food intake or nutritional requirements with advancing age with either social factors (e.g. loss of a partner) or physiological changes. The social change was most commonly the adjustment to living alone and cooking for one, which impacted on quantity of food consumed. There was also recognition that a reduced appetite was associated with lower activity levels and that keeping physically active could improve appetite.

"And the fact that you live on your own and you're not cooking. My husband had an enormous appetite, and of course you know you're cooking for two, and you sit down and you're talking, you do eat more." (Female \# 7, 86 years)

Physiological changes included alterations in taste, appetite or metabolic changes resulting in smaller food portions consumed. Although participants often reported that their appetite was good, it was generally felt that it had declined with age.

"We've cut down .... we used to have a piece of steak you know oh it'd be bigger than that but we, now we would only have half a scotch fillet each." (Female \#2, 83 years)

\section{Dietary advice or assistance}

\section{GP first point of contact}

Most participants identified their general practitioner (GP) as the first point of contact if they had any dietary concerns. They trusted the doctor to tell them if there was any need to alter their diet and to answer any questions they had. Two participants felt that their doctor would refer them to a dietitian if required. Family, friends and the media were also sources of dietary information.

In terms of receiving assistance with services such as home delivered meals (only one participant was occasionally using a home delivered meal service), they were considered a possibility but the preference was to have home prepared meals. There was a focus on consuming fresh or home-made meals.

"But any food that had to have been cooked and frozen and then delivered, it's just not like fresh food." (Female \#8, 78 years)

\section{Discussion}

This study aimed to build on our understanding of what influences food choices and dietary patterns of adults over 75 years of age in Australia. We found that participants placed a high value on eating well and their food choices were driven by childhood eating patterns, and their specific health conditions which frequently resulted in self-imposed dietary restrictions. Age related changes were seen as inevitable and could be divided into physiological changes such as reduced appetite or social changes such as living alone. The first option for seeking dietary advice was the GP, and while services such as home delivered meals were considered acceptable, freshly prepared meals were the preferred option.

The participants in this study were living independently with very few support services, and the majority were still able to drive. Although all but one were living alone they placed a high value on continuing to eat well and preparing meals for themselves. Vesnaver and colleagues described a model of 'dietary resilience' based on interviews with 30 Canadian adults aged between 73 and 87 years (22). One of the features of dietary resilience was prioritizing eating well, enabling individuals to adapt and overcome dietary obstacles. This notion of resilience is consistent with the themes we identified of independence and value of eating well where, despite being faced with challenges, food intake was maintained.

Routine and childhood meal patterns were contributing factors to current dietary practices and this has also been identified in other older populations. A study of Scottish adults aged 75 years and older used 24 hour food recall in conjunction with interviews to understand dietary beliefs and practices (18). They found routine was seen as an important way of overcoming fluctuations in appetite, and the establishment of dietary beliefs and habits in childhood carried over into old age.

The issue of weight management and dietary restriction is an important area to explore further. We found that management of weight was a common area of concern for participants, as it had been a main focus of their diet during adulthood. However, in older adults, a higher BMI is associated with lower mortality (23), and 
weight change is associated with greater mortality (24). In addition to weight concerns, a number of other dietary restrictions had been adopted without any specific guidance, including reduced fat, reduced lactose, reduced fructose and avoidance of certain additives. Dietary restrictions in older people are considered to have an unfavourable benefit / risk ratio with the potential to result in deficiencies and contribute to under-nutrition $(7,25)$. Further investigation is required to determine whether these restrictive practices have an impact on nutritional adequacy in this population.

Age-related changes impacting on food intake such as reduced appetite, social isolation, altered capacity to shop and prepare food have been well described in the literature (26). Although the participants in this study did identify changes in appetite, reduced serve sizes, and issues associated with living alone and cooking for one they tended to downplay these factors and felt that they were inevitable part of aging that weren't impacting on their overall nutritional intake. Ramic and colleagues have shown that living alone for older adults was associated with reduced nutrient intake, reduced $\mathrm{BMI}$ and greater nutritional risk, however those living alone were also more financially compromised (27). Participants in our study were generally unconcerned with changes to appetite or portion sizes and appeared unaware of any specific changes to their nutritional requirements with age (such as needing additional protein or calcium). It may be that nutrition messages for older adults need to address how to meet their needs in the face of changing dietary patterns in order to maintain optimal health.

The clearest source of dietary advice, if required, was identified as the GP consistent with other studies which have identified GPs as a trusted source of information $(17,28)$. In Australia, there are no guidelines on managing nutritional issues for older adults, particularly the frail elderly and therefore GPs may not be fully informed on the specific requirements of this population and unable to provide appropriate guidance.

Our study has limitations in that the sample was predominantly women who were generally well and independent. They exhibited traits of 'dietary resilience' but further exploration of the issues with a male population would provide additional insights. Literature suggests that older men living alone tend to have poorer cooking skills, associated with a poorer quality diet $(29,30)$ and may be more affected by changes to living situation. A recent literature review suggests that there may be gender differences in the impact of living alone on food intake, with men more likely to show undesirable intakes (31). It would also be useful to compare our findings with a malnourished, frailer population to understand the influences on their food choices.

This sample of older adults placed high value on eating well as they age, however a number continued with dietary restrictions which have the potential to compromise their nutrition as dietary requirements change. Further research is needed into how to communicate changing nutritional needs to this group and to determine whether primary care staff are equipped to provide appropriate nutrition information.

Acknowledgements: The authors would like to thank Kate Wingrove for her invaluable assistance in coding a sample of the interviews. We would also like to thank the staff at the medical centre for their role in recruiting participants, co-ordinating interview times and providing interview facilities. And finally, we would like to thank the participants for their willingness to provide their time for the project.

Conflict of interest: Ms Winter reports other from Nestle Health Science (employee of the company), outside the submitted work. Dr. McNaughton has nothing to disclose. Dr. Nowson reports grants from Nestle Health Science, grants and personal fees from Meat and Livestock Australia, personal fees from Dairy Health Nutrition Consortium outside the submitted work and is a member of AWASH and WASH (Australian Division of World Action on Salt and Health) but does not receive any financial support from these organisations..

Ethical Standards: Study protocol approved by Deakin University Human Research Advisory Committee.

\section{References}

1. Donini LM, Scardella P, Piombo L, et al. Malnutrition in elderly: social and economic determinants. J Nutr Health Aging 2013;17:9-15.

2. Morley JE. Anorexia of aging: physiologic and pathologic. Am J Clin Nutr 1997;66:760-73.

3. de Groot CPGM, van Staveren WA. Undernutrition in the European SENECA studies. Clin Geriatr Med 2002;18:699.

4. De Castro JM. Age-related changes in spontaneous food intake and hunger in humans. Appetite. 1993;21:255-72.

5. Australian Bureau Statistics. Australian Health Survey 2011-2013. Available from: http: / / www.abs.gov.au/australianhealthsurvey. Accessed 23 August, 2015

6. Vesnaver E, Keller HH. Social influences and eating behavior in later life: a review. J Nutr Gerontol Geriatr 2011;30:2-23.

7. Darmon P, Kaiser MJ, Bauer JM, Sieber CC, Pichard C. Restrictive diets in the elderly: Never say never again? Clin Nutr 2010;29:170-4

8. Anyanwu UO, Sharkey JR, Jackson RT, Sahyoun NR. Home food environment of older adults transitioning from hospital to home. J Nutr Gerontol Geriatr 2011;30:105-21.

9. Sharkey JR. Nutrition risk screening: the interrelationship of food insecurity, food intake, and unintentional weight change among homebound elders. J Nutr Elderly. 2004;24:19-34

10. Odlund Olin A, Koochek A, Ljungqvist O, Cederholm T. Nutritional status, well-being and functional ability in frail elderly service flat residents. Eur J Clin Nutr. 2004;59:263-70.

11. Bartali B, Frongillo E, Bandinelli S, et al. Low nutrient intake is an essential component of frailty in older persons. J GerontolSeries A: Biolog Med Sci 2006;61:589.

12. Winter J, Flanagan D, McNaughton SA, Nowson C. Nutrition screening of older people in a community general practice, using the MNA-SF. J Nutr Health Aging. 2013;17:322-5.

13. Rist G, Miles G, Karimi L. The presence of malnutrition in community-living older adults receiving home nursing services. Nutr Diet. 2012;69:46-50.

14. Flanagan D, Fisher T, Murray M, Visvanathan R, Charlton K, Thesing C, et al. Managing undernutrition in the elderly - prevention is better than cure. Aust Fam Physician. 2012;41:695-9.

15. Leggo M, Banks M, Isenring E, Stewart L, Tweeddale M. A quality improvement nutrition screening and intervention program available to Home and Community Care eligible clients. Nutr Diet 2008;65:162-7.

16. Charlton KE, Walton K, Moon L, Smith K, McMahon AT, Ralph F, et al. «It could probably help someone else but not me»: a feasibility study of a snack programme offered to meals on wheels clients. J Nutr Health Aging. 2013;17:364-9.

17. Ball L, Desbrow B, Leveritt M. An exploration of individuals' preferences for nutrition care from Australian primary care health professionals. Aust J Prim Health. 2014;20:113-20.

18. McKie L, MacInnes A, Hendry J, Donald S, Peace H. The food consumption patterns and perceptions of dietary advice of older people. J Hum Nutr Diet 2000;13:173-83.

19. Swift JA, Tischler V. Qualitative research in nutrition and dietetics: getting started. J Hum Nutr Diet 2010;23:559-66.

20. Burnard P. A method of analysing interview transcripts in qualitative 
research. Nurse Educ Today. 1991;11:461-6.

21. Braun V, Clarke V. Using thematic analysis in psychology. Qual Res Psychol. 2006;3:77-101.

22. Vesnaver E, Keller HH, Payette H, Shatenstein B. Dietary resilience as described by older community-dwelling adults from the NuAge study «if there is a will -there is a way!». Appetite 2012;58:730-8.

23. Winter JE, MacInnis RJ, Wattanapenpaiboon N, Nowson CA. BMI and allcause mortality in older adults: a meta-analysis. Am J Clin Nutr 2014;99:87590.

24. Somes GW, Kritchevsky SB, Shorr RI, Pahor M, Applegate WB. Body mass index, weight change, and death in older adults: the systolic hypertension in the elderly program. Am J Epidemiol 2002;156:132-8.

25. Zeanandin G, Molato O, Le Duff F, Guérin O, Hébuterne X, Schneider SM. Impact of restrictive diets on the risk of undernutrition in a free-living elderly population. ClinNutr 2012;31(1):69-73.

26. de Boer A, Ter Horst GJ, Lorist MM. Physiological and psychosocial agerelated changes associated with reduced food intake in older persons. Ageing
Res Rev. 2013;12(1):316-28.

27. Ramic E, Pranjic N, Batic-Mujanovic O, Karic E, Alibasic E, Alic A. The effect of loneliness on malnutrition in elderly population. Medicinski Arhiv. 2011;65(2):92-5.

28. Cash T, Desbrow B, Leveritt M, Ball L. Utilization and preference of nutrition information sources in Australia. Health Expectations: An International Journal Of Public Participation In Health Care And Health Policy. 2014.

29. Hughes G, Bennett KM, Hetherington MM. Old and alone: barriers to healthy eating in older men living on their own. Appetite. 2004;43(3):269-76.

30. Charlton KE. The nutrient intake of elderly men living alone and their attitudes towards nutrition education. Abbreviate title to J Hum Nutr Diet. 1997;10(6):343-52.

31. Hanna KL, Collins PF. Relationship between living alone and food and nutrient intake. Nutr Rev 2015;73(9):594-611. 